\title{
Total testosterone to dihydrotestosterone ratio assessed by LC-MS/MS predicts a worse metabolic profile not only in PCOS patients
}

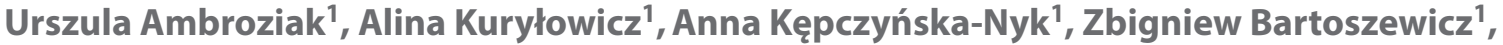 \\ Agnieszka Kondracka' ${ }^{1}$, Radosław Jaźwiec ${ }^{2}$, Emilia Samborowska², Michał Dadlez², \\ Aleksandra Wysłouch-Cieszyńska², Tomasz Bednarczuk ${ }^{1}$ \\ ${ }^{1}$ Department of Internal Medicine and Endocrinology, Medical University of Warsaw, Poland \\ ${ }^{2}$ Mass Spectrometry Laboratory, Institute of Biochemistry and Biophysics, Polish Academy of Sciences, Warsaw, Poland
}

\begin{abstract}
Objectives: Total testosterone/dihydrotestosterone ratio (TT/DHT) was found to determine metabolic risk in polycystic ovary syndrome (PCOS). The aim of this study was to analyze whether (TT/DHT) may be helpful in predicting metabolic risk not only in PCOS patients but also in healthy women.

Material and methods: Total testosterone (TT), dihydrotestosterone (DHT), androstendione and dehydroepiandrosterone sulphate (DHEA-S) were measured by LC-MS/MS in 36 women with PCOS and in 29 age-matched controls without clinical hyperandrogenism. In all participants, anthropometric data, lipids, adipose tissue percent (\%fat), HOMA-IR were also assessed. Results: The studied groups were not different in terms of age, BMI, waist circumference, \%fat and HOMA-IR. In the patients group, mean TT and androstendione levels were significantly higher as compared to controls $(1.4 \mathrm{nmol} / \mathrm{L} \mathrm{vs.} 1.0 \mathrm{nmol} / \mathrm{L}$, $\mathrm{P}<0.001)$ and $(6.6 \mathrm{nmol} / \mathrm{L}$ vs. $4.9 \mathrm{nmol} / \mathrm{L}, \mathrm{P}<0.01)$, respectively. In the patients group, mean TT/DHT ratio was significantly higher compared to controls (3.6 vs. 2.7, $\mathrm{P}<0.01)$ and correlated with BMI $(r=0.37, \mathrm{P}<0.05)$, waist circumference $(r=0.44$, $P<0.01)$, \%fat $(r=0.30, P<0.05)$, as well as with insulin levels $(r=0.38, P<0.05)$ and HOMA-IR $(r=0.44, P<0.05)$. The association between TT/DHT ratio and unfavorable metabolic parameters was also seen in controls.

Conclusion: Total testosterone/dihydrotestosterone ratio assessed by LC-MS/MS correlates with a worse metabolic profile not only in PCOS patients, but also in healthy women.
\end{abstract}

Key words: testosterone, dihydrotestosterone, liquid chromatography/mass spectrometry, polycystic ovary syndrome, hyperandrogenism

Ginekologia Polska 2017; 88, 1:5-8

\section{INTRODUCTION}

Polycystic ovary syndrome (PCOS) represents the most common cause of hyperandrogenism. Based on the clinical picture and laboratory findings, three main phenotypes of PCOS can be defined: metabolic, hyperandrogenic and reproductive [1]. Women with PCOS demonstrate a significantly higher prevalence of impaired glucose tolerance and noninsulin dependent diabetes, hyperlipidemia, hypertension, carotid and coronary atherosclerosis, and endothelial dysfunction compared with age- and weight-matched control subjects, which determine increased cardiovascular risk in this group of patients [2,3]. Health providers should focus on prophylactic activities, especially in the subgroup of PCOS women at higher risk of metabolic complications. Therefore, there is the need to identify predictors that would help to identify PCOS patients with the highest metabolic risk. Münzker et al. proposed total testosterone/dihydrotestosterone ratio (TT/DHT) as an indicator of a worse metabolic prognosis in PCOS [4]. Metabolic disturbances observed in patients with PCOS result from the unfavorable influence of androgens on glucose and lipid metabolism as well as on insulin action. In turn, hyperinsulinemia stimulates androgens 
synthesis in ovaries, closing the vicious circle [5]. Therefore, we have aimed at analyzing whether (TT/DHT) may be helpful in predicting metabolic risk not only in PCOS patients but also in healthy women not meeting PCOS criteria.

As liquid chromatography coupled with mass spectrometry (LC-MS/MS) is indicated as the method with the highest accuracy, we have used this method to measure androgens concentrations in order to look for relations between androgen levels, TT/DHT ratio and the metabolic profile in PCOS patients and controls $[6,7]$.

\section{MATERIAL AND METHODS}

\section{Subjects}

36 women (18-36 years old, mean age 25.3) with PCOS were consecutively recruited from the Department of Internal Medicine and Endocrinology, Medical University of Warsaw, between June 2012 and February 2014. The diagnosis was established based on Rotterdam ESHRE criteria [1]. The control group consisted of 29 healthy women aged 19-45 years (mean age 27.9) with regular menses, without clinical hyperandrogenism (acne and/or androgenic alopecia and/or hirsutism and/or virilization). Exclusion criteria for both groups were as follows: age $<18$ years or $>45$ years, pregnancy, recent history (up to 3 months) of hormonal contraceptive or hormonal replacement therapy use. The group was partially described previously [8].

All participants have undergone clinical and hormonal assessment. Clinical evaluation was based on anthropometric measurements (including weight, height, waist circumference (WC) and assessment of fat tissue content by the bioelectrical impedance method), the data on menstruation regularity, presence of clinical hyperandrogenism (hirsutism, acne, androgenic alopecia, virilization). Oligoovulation/anovulation was defined by oligomenorrhoea/amenorrhoea. Hirsutism was defined by a Ferriman-Gallwey score $\geq 8$, presence of acne and alopecia was recorded but not scored [9]. Morphology of ovaries was assessed by pelvic ultrasound.

\section{Biochemical measurements}

Laboratory tests consisted of a panel of simultaneously measured serum hormones including total testosterone (TT), dihydrotestosterone (DHT), dehydroepiandrosterone sulphate (DHEA-S) and androstendione as well as sex hormone binding globulin (SHBG), albumin, fasting glucose, insulin and lipids.

The blood samples for hormonal assessment were collected in the morning (7:00-9:00 am) during the early follicular phase of the menstrual cycle ( $3^{\text {th }}-5^{\text {th }}$ day).

Analysis of albumin concentrations was performed by Cobas ${ }^{\circledR}$ diagnostic kits (Roche Diagnostics $\mathrm{GmbH}$, Mannheim, Germany), SHBG by ELISA DRG ${ }^{\circ}$ (DRG Instruments $\mathrm{GmbH}$, Marburg, Germany), while serum TT, DHT, androstendione,
DHEA-S by LC-MS/MS. Free androgen index (FAI) was calculated according to the formula (TTx100/SHBG).

LC-MS/MS measurements were performed in the Mass Spectrometry Laboratory of the Institute of Biochemistry and Biophysics, Polish Academy of Sciences. Samples were analyzed using Absolute IDQ Stero17 Kit produced by Biocrates Life Sciences AG (Innsbruck, Austria) according to the validated protocol supplied by the manufacturer. Steroids were analyzed in positive ionization (for DHEA-S both positive and negative) in multiple reaction monitoring mode [10]. The intra- and interassay variability did not exceed $11.5 \%$.

The project has been approved by the Bioethical Committee of the Medical University of Warsaw, and written informed consent for participation in this study was obtained from all participants.

\section{Statistical analyses}

The differences in androgens concentrations were assessed with Statistica software package v.10 (StatSoft, Tulsa, OK, USA), using paired t test. All correlations between quantitative values were performed with the Pearson correlation test. For all tests, the level of significance was established at 0.05 .

\section{RESULTS}

The basic clinical characteristics of PCOS patients as well as the control group are summarized in Table 1.

\section{TT/DHT ratio}

Mean TT/DHT ratio in the patients group was significantly higher as compared to controls: 3.6 (1.4-7.6) and 2.7 (1.2-4.9), respectively, $\mathrm{P}<0.01$.

\section{TT/DHT ratio correlations}

In the patients group, TT/DHT ratio correlated positively with anthropometric parameters: $\mathrm{BMI}(\mathrm{r}=0.37, \mathrm{P}<0.05)$, WC $(r=0.44, P<0.01)$, \%fat $(r=0.30, P<0.05)$ as well as with insulin level $(r=0.38, P<0.05)$ and HOMA-IR $(r=0.44, P<0.05)$.

The positive correlations between TT/DHT and BMI $(r=0.50, P<0.01)$, WC $(r=0.5, P<0.01)$, \%fat $(r=0.64, P<0.001)$, insulin level $(r=0.35, P<0.05)$ and HOMA-IR $(r=0.41, P<0.05)$ were also present in the healthy subjects group.

The differences in TT/DHT ratio in terms of BMI, WC, \%fat and HOMA-IR are presented in Table 2. The ratio was significantly higher in PCOS patients and controls, in subgroups with greater WC, \%fat and HOMA-IR. In controls, significantly greater TT/DHT was also detected in terms of BMI.

\section{Androgens (total testosterone, FAI, DHT, androstendione, DHEA-S)}

Mean TT concentration in the patients group $(1.4 \mathrm{nmol} / \mathrm{L}$, 0.7-3.1) was significantly higher as compared to controls $(1.0 \mathrm{nmol} / \mathrm{L}, 0.3-1.4), \mathrm{P}<0.001$. 
Table 1. Characteristics of studied groups

\begin{tabular}{|c|c|c|c|}
\hline & $\begin{array}{l}\text { Patients with } \mathrm{HA} \\
\qquad(\mathrm{N}=36)\end{array}$ & $\begin{array}{l}\text { Controls } \\
(\mathrm{N}=29)\end{array}$ & $\mathbf{P}$ \\
\hline Age, years, mean $( \pm S D)$ & $25.3( \pm 4.7)$ & $27.9( \pm 7.5)$ & 0.1 \\
\hline $\mathrm{BMI}, \mathrm{kg} / \mathrm{m}^{2}$, mean $( \pm \mathrm{SD})$ & $25.3( \pm 7.3)$ & $23.3( \pm 5.5)$ & 0.4 \\
\hline Waist circumference, $\mathrm{cm}$, mean $( \pm S D)$ & $77.4( \pm 14.2)$ & $76.2( \pm 10.9)$ & 0.8 \\
\hline$\%$ of adipose tissue* mean $( \pm$ SD) & $32.9( \pm 8.5)$ & $29.1( \pm 6.8)$ & 0.1 \\
\hline Insulin, $\mu \mathrm{IU} / \mathrm{mL}$, mean $( \pm \mathrm{SD})$ & $7.4( \pm 3.2)$ & $6.8( \pm 4)$ & 0.9 \\
\hline HOMA-IR, mean ( \pm SD) & $1.6( \pm 0.7)$ & $1.57( \pm 0.9)$ & 0.7 \\
\hline $\mathrm{HDL}-\mathrm{C}, \mathrm{mmol} / \mathrm{L}$, mean $( \pm \mathrm{SD})$ & $2.3( \pm 1.3)$ & $2.4( \pm 0.7)$ & 0.3 \\
\hline Triglicerydes, mmol/L, mean ( \pm SD) & $0.6( \pm 0.2)$ & $0.7( \pm 0.1)$ & 0.7 \\
\hline Metabolic syndrome, $\mathrm{n}$ & 2 & 3 & 0.9 \\
\hline Ferriman-Gallwey score, mean $( \pm S D)$ & $4.7( \pm 5)$ & $0.59( \pm 1.6)$ & $<0.0001$ \\
\hline Acne, $n(\%)$ & $24(66.6)$ & 0 & $<0.0001$ \\
\hline Menstrual disturbances, $\mathrm{n}(\%)$ & $25(69.4)$ & 0 & $<0.0001$ \\
\hline Total testosterone, $\mathrm{nmol} / \mathrm{L}$, mean $( \pm \mathrm{SD})$ & $1.4( \pm 0.7)$ & $1.0( \pm 0.3)$ & $<0.001$ \\
\hline $\mathrm{FAl}$, mean $( \pm \mathrm{SD})$ & $2.2( \pm 1.1)$ & $1.7( \pm 1.0)$ & $<0.05$ \\
\hline $\mathrm{DHT}$, nmol/L, mean ( $\pm \mathrm{SD})$ & $0.3( \pm 0.3)$ & $0.3( \pm 0.3)$ & 0.7 \\
\hline Androstendione, $\mathrm{nmol} / \mathrm{L}$, mean $( \pm \mathrm{SD})$ & $6.6( \pm 2.1)$ & $4.9( \pm 1.7)$ & $<0.01$ \\
\hline DHEA-S, $\mu \mathrm{mol} / \mathrm{L}$, mean $( \pm \mathrm{SD})$ & $7.6( \pm 2.4)$ & $6.9( \pm 2.5)$ & 0.2 \\
\hline
\end{tabular}

*Measured by the bioelectrical impedance method

$\mathrm{BMI}$ — body mass index, defined as the body mass $(\mathrm{kg})$ divided by the square of the body height $(\mathrm{m})$; DHEA-S — dehydroepiandrosterone sulphate;

$\mathrm{DHT}$ - dihydrotestosterone; FAI — free androgen index; HA - hyperandrogenism; HDL-C — high density lipoprotein cholesterol; HOMA-IR - insulin resistance index; $\mathrm{n}$ - number

Table 2. Differences in TT/DHT (total testosterone/dihydrotestosterone) ratio in PCOS patients and controls in terms of BMI, WC, HOMA-IR and $\%$ of adipose tissue

\begin{tabular}{|l|c|c|c|c|c|c|}
\hline Parameter & Ranges & PCOS & P & Controls & P** \\
\hline BMI $\left[\mathrm{kg} / \mathrm{m}^{2}\right]$ & $\geq 30$ vs. $<30$ & $4.3 \pm 1.3$ vs. $3.4 \pm 1.3$ & 0.1 & $4.3 \pm 0.4$ vs. $2.6 \pm 1.1$ & 0.047 \\
\hline WC $[\mathrm{cm}]$ & $>80$ vs. $\leq 80$ & $4.4 \pm 0.9$ vs. $3.3 \pm 1.4$ & 0.01 & $3.9 \pm 0.6$ vs. $2.5 \pm 1.1$ & 0.03 \\
\hline$\%$ of adipose tissue* & $\geq 30$ vs. $<30$ & $4.9 \pm 1.5$ vs. $3.2 \pm 1.1$ & 0.009 & $3.6 \pm 1.5$ vs. $2.4 \pm 1.0$ & 0.0002 \\
\hline HOMA-IR & $\geq 2$ vs. $<2$ & $4.2 \pm 1.5$ vs. $2.9 \pm 0.9$ & 0.01 & $3.7 \pm 0.9$ vs. $2.0 \pm 0.6$ & 0.11 \\
\hline
\end{tabular}

*Measured by the bioelectrical impedance method; ** PCOS vs. controls

$\mathrm{BMI}$ - body mass index; WC - waist circumference; HOMA-IR — insulin resistance index

In both groups, TT levels correlated positively with HDL-C ( $r=0.37, \mathrm{P}<0.05)$, while no correlations with BMI, WC, \%FAT, HOMA-IR as well as insulin, total cholesterol (TC) and TG levels were detected.

FAl in the patients group correlated positively with BMI $(r=0.35, P<0.05)$, WC $(r=0.33, P<0.05)$, \%FAT $(r=0.386$, $P<0.05)$, HOMA-IR $(r=0.47, P<0.01)$ and insulin level $(r=0.43$, $\mathrm{P}<0.05)$. In the control group, only the relation with insulin $(r=0.57, P<0.01)$ and HOMA-IR $(r=0.59, P<0.01)$ was present.

Mean DHT concentration in the patients group, $0.3 \mathrm{nmol} / \mathrm{L}$ (0.3-1.4), was not different from controls, $0.3 \mathrm{nmol} / \mathrm{L}$ (0.3-1.0). No correlations between DHT levels and metabolic parameters (BMI, \%FAT, insulin, HOMA-IR, TC, HDL-C and TG) were detected. Interestingly, DHT correlated negatively with WC $(r=-0.37, \mathrm{P}<0.05$ - the higher WC, the lower DHT).
Mean androstendione concentration in the patients group was significantly higher, $6.6 \mathrm{nmol} / \mathrm{L}(3.1-12.2)$, as compared to controls, $4.9 \mathrm{nmol} / \mathrm{L}$ (1.4-10.1), $\mathrm{P}<0.01$.

Mean DHEA-S concentration did not differ significantly between the studied groups. No correlation between androstendione and DHEA-S levels and metabolic parameters was detected in either studied group.

\section{DISCUSSION}

The results of our study showed that TT/DHT ratio correlated with a worse metabolic profile not only in PCOS patients but also in healthy subjects without hyperandrogenism. Our finding suggests that TT/DHT ratio may speak for metabolic disturbance, not only in PCOS but also in "theoretically" healthy subjects without clinical and hormo- 
nal hyperandrogenism. Moreover, increased TT/DHT ratio may be considered as a predictor of development of PCOS in this group of women in future.

Ollila et al. in their large study concluded that symptoms or diagnosis of PCOS are associated with dyslipidemia and significantly increased weight gain, especially in early adulthood [11]. Subsequently, a number of studies were conducted in order to identify potential predictors of the metabolic risk in this group of patients. Valderhaug et al. observed that among obese women, not meeting PCOS criteria, those with hyperandrogenemia have a worse metabolic phenotype [12]. In other studies, a worse metabolic profile in PCOS patients was associated with higher testosterone concentrations [13]. In turn, Li et al. showed that higher FAl in patients with PCOS correlates with higher BMI, \%fat, TC, DHEA-S and HOMA-IR [14]. Recently, Münzker et al. suggested that increased TT/DHT ratio is a good predictor of unfavorable metabolic profile in this group of patients [4]. The results of our study confirm this hypothesis but also suggest that high TT/DHT ratio can be considered as a risk factor of metabolic disturbances in women without signs or symptoms of PCOS. What is important, the control group investigated in our study was not only BMI-matched but also did not differ from PCOS patients in terms of WC, content of adipose tissue, insulin level and HOMA-IR. In the study of Münzker et al., the control group differed from the PCOS patients by mean insulin levels and HOMA-IR [4].

In contrast to TT/DHT ratio, in our control group no correlation between TT alone and metabolic parameters was found, while we found a strong relation between FAI and insulin/HOMA-IR. This finding can be explained by the influence of insulin on SHBG level [15]. Moreover, we have not observed an association between other androgens levels and metabolic profile either in controls or in PCOS patients, and this finding is consistent with the results of others [16].

The undeniable strength of our study was the application of the LC-MS/MS method for steroid measurements that assures high accuracy of the obtained results. Another strength is careful selection of the control group that was not different from the PCOS patients in terms of anthropometric parameters as well as insulin concentration and HOMA-IR. However, due to the relatively small size of the investigated groups, further studies and larger trials are needed to verify our results.

\section{CONCLUSIONS}

TT/DHT ratio correlates with a worse metabolic profile in PCOS patients but also in healthy women.

\section{REFERENCES}

1. Rotterdam ESHRE/ASRM-Sponsored PCOS Consensus Workshop Group. Revised 2003 consensus on diagnostic criteria and long-term health risks related to polycystic ovary syndrome. Fertil. Steril. 2004; 81(1): 19-25, doi: 10.1093/humrep/deh098, indexed in Pubmed: 14711538.

2. Randeva HS, Tan BK, Weickert MO, et al. Cardiometabolic aspects of the polycystic ovary syndrome. Endocr. Rev. 2012; 33(5): 812-841, doi: 10.1210/er.2012-1003, indexed in Pubmed: 22829562.

3. Mani H, Levy MJ, Davies MJ, et al. Diabetes and cardiovascular events in women with polycystic ovary syndrome: a 20-year retrospective cohort study. Clin. Endocrinol. (Oxf). 2013; 78(6): 926-934, doi: 10.1111/cen.12068, indexed in Pubmed: 23046078.

4. Münzker J, Hofer D, Trummer C, et al. Testosterone to dihydrotestosterone ratio as a new biomarker for an adverse metabolic phenotype in the polycystic ovary syndrome. J. Clin. Endocrinol. Metab. 2015; 100(2): 653-660, doi: 10.1210/jc.2014-2523, indexed in Pubmed: 25387259.

5. Shroff R, Syrop CH, Davis W, et al. Risk of metabolic complications in the new PCOS phenotypes based on the Rotterdam criteria. Fertil. Steril. 2007; 88(5): 1389-1395, doi: 10.1016/j.fertnstert.2007.01.032, indexed in Pubmed: 17462641.

6. Stanczyk FZ, Clarke NJ. Advantages and challenges of mass spectrometry assays for steroid hormones. J. Steroid Biochem. Mol. Biol. 2010; 121(3-5): 491-495, doi: 10.1016/j.jsbmb.2010.05.001, indexed in Pubmed: 20470886

7. Honour JW. 17-Hydroxyprogesterone in children, adolescents and adults. Ann. Clin. Biochem. 2014; 51(Pt 4): 424-440, doi: 10.1177/ /0004563214529748, indexed in Pubmed: 24711560.

8. Ambroziak U, Kępczyńska-Nyk A, Kuryłowicz A, et al. LC-MS/MS improves screening towards 21-hydroxylase deficiency. Gynecol. Endocrinol. 2015; 31(4): 296-300, doi: 10.3109/09513590.2014.994599, indexed in Pubmed: 25539143.

9. Ferriman D, Gallwey JD. Clinical assessment of body hair growth in women. J. Clin. Endocrinol. Metab. 1961; 21: 1440-1447, doi: 10.1210/jcem21-11-1440, indexed in Pubmed: 13892577.

10. Koal T, Schmiederer D, Pham-Tuan H, et al. Standardized LC-MS/MS based steroid hormone profile-analysis.J. Steroid Biochem. Mol. Biol. 2012; 129(3-5): 129-138, doi: 10.1016/j.jsbmb.2011.12.001, indexed in Pubmed: 22210511.

11. Ollila MME, Piltonen T, Puukka K, et al. Weight Gain and Dyslipidemia in Early Adulthood Associate With Polycystic Ovary Syndrome: Prospective Cohort Study. J. Clin. Endocrinol. Metab. 2016; 101(2): 739-747, doi: 10.1210/jc.2015-3543, indexed in Pubmed: 26652764

12. Valderhaug TG, Hertel JK, Nordstrand N, et al. The association between hyperandrogenemia and the metabolic syndrome in morbidly obese women. Diabetol Metab Syndr. 2015; 7: 46, doi: 10.1186/s13098-0150040-5, indexed in Pubmed: 26015809.

13. Moran L, Teede H. Metabolic features of the reproductive phenotypes of polycystic ovary syndrome. Hum. Reprod. Update. 2009; 15(4): 477-488, doi: 10.1093/humupd/dmp008, indexed in Pubmed: 19279045.

14. Li $H, X u X$, Wang $X$, et al. Free androgen index and Irisin in polycystic ovary syndrome. J. Endocrinol. Invest. 2016; 39(5): 549-556, doi: 10.1007/s40618-015-0403-7, indexed in Pubmed: 26584566.

15. Akin F, Bastemir M, Kaptanoglu B. Relationship between insulin and sex hormone-binding globulin levels during weight loss in obese women. Ann. Nutr. Metab. 2007;51(6):557-562, doi: 10.1159/000114210, indexed in Pubmed: 18227624.

16. Lerchbaum E, Schwetz V, Rabe T, et al. Hyperandrogenemia in polycystic ovary syndrome: exploration of the role of free testosterone and androstenedione in metabolic phenotype. PLoS ONE. 2014; 9(10): e108263, doi: 10.1371/journal.pone.0108263, indexed in Pubmed: 25310562. 